\title{
A Markov-Random-Field Approach for Extracting Straight-Line Segments of Roofs From High-Resolution Aerial Images
}

\author{
Vanessa Jordão Marcato Fernandes and Aluir Porfírio Dal Poz
}

\begin{abstract}
This paper proposes a method for extracting groups of straight lines that represent roof boundary sides and roof ridgelines from high-resolution aerial images using corresponding airborne laser scanner (ALS) roof polyhedrons as initial approximations. Our motivation for this research is the possibility of future use of resulting image-space straight lines in several applications. For example, straight lines that represent roof boundary sides and precisely extracted from a high-resolution image can be back-projected onto the ALS-derived building polyhedron for refining the accuracy of its boundary. The proposed method is based on two main steps. First, straight lines that are candidates to represent roof ridgelines and roof boundary sides of a building are extracted from the aerial image. The ALS-derived roof boundary sides and roof ridgelines are projected onto the image space, and bolding boxes are constructed around the projected straight lines while considering the projection errors. This allows the extraction of straight lines within the bounding boxes. Second, a group of straight lines that represent roof boundary sides and roof ridgelines of a selected building is obtained through the optimization of a Markov random field-based energy function using the genetic algorithm optimization method. The formulation of this energy function considers several attributes, such as the proximity of the extracted straight lines to the corresponding projected ALS-derived roof polyhedron and the rectangularity (extracted straight lines that intersect at nearly $90^{\circ}$ ). In order to validate the proposed method, four experiments were accomplished using high-resolution aerial images, along with interior and exterior orientation parameters, and available ALS-derived building roof polyhedrons. The obtained results have shown that the method works properly and this will be qualitatively and quantitatively demonstrated in this research.
\end{abstract}

Index Terms-Airborne laser scanner (ALS), Markov random field (MRF), straight line.

\section{INTRODUCTION}

B UILDING extraction methods are important in the context of capturing and updating spatial data for applications that involve urban areas. For example, accurate and reliable building roof boundaries are useful in applications that involve real estate, large-scale mapping, and risk management. However,

Manuscript received March 7, 2016; revised June 11, 2016; accepted August 2, 2016. Date of publication September 26, 2016; date of current version November 30, 2016. This work was supported in part by FAPESP (São Paulo State Foundation for Scientific Research) under Grant 2013/13138-0 and Grant 2012/22332-2, and in part by CNPq (National Council for Scientific and Technological Development, Brazil) under Grant 304879/2009-6. (Corresponding author: Vanessa Jordão Marcato Fernandes.)

V. J. M. Fernandes is with the São Paulo State University, Presidente Prudente 19060-900, Brazil (e-mail: vanessamarcato@yahoo.com.br).

A. P. Dal Poz is with the Department of Cartography, São Paulo State University, Presidente Prudente 19060-900, Brazil (e-mail: aluir@fct.unesp.br).

Color versions of one or more of the figures in this paper are available online at http://ieeexplore.ieee.org.

Digital Object Identifier 10.1109/JSTARS.2016.2601068 automated building roof extraction has remained a challenging task, mainly due to the varying building roof configurations, the varying neighborhood contexts, and noise in the input data. High-resolution images are rich in semantic information and in fine geometric details, but they can be affected by illumination differences and occlusions. However, the airborne laser scanner (ALS) directly provides three-dimensional (3-D) point clouds with high confidence height information, which is the most useful data for detecting aboveground objects. However, ALS data are poor in semantic information and, due to the inherent subsampling process, are also poor in geometric details. Due to the complementary characteristics of these two data sources, it is expected that objects, such as buildings, can be extracted with more accuracy, completeness, and reliability than by using a single data source.

Gilani et al. [1] presented a commonly used criterion for classifying building extraction methods that is based on the input data, such as methods that use ALS data, image data, and combinations of ALS and image data. Image-based building extraction methods exploit 2-D information that is derived from a single image or 3-D information that is derived from stereoscopic images. This category of methods has been used since the 1980s. Fua and Hanson [2] presented a method for outlining complex rectilinear buildings in aerial images. Müller and Zaum [3] used a region-growing segmentation method and a classification procedure for detecting buildings in aerial images. Akçay and Aksoy [4] combined spectral and structural information to separate buildings from other objects, and Ferraioli [5] used a Markov random field (MRF) for building edge detection. Sirmaçek and Ünsalan [6] presented a probabilistic approach for detecting buildings in aerial and satellite images.

ALS-based methods usually exploit the advantage of directly using the height discontinuity to detect building points. Due to this, ALS data provide a higher level of automation than imagebased methods [7]. However, because ALS points are subsampled, building roof boundaries cannot be precisely extracted [8]. The precision of extracted building roof boundaries depends on the density of the ALS point cloud, i.e., the denser the ALS data, the more precise the extracted building roof boundaries. Methods for building detection or extraction from ALS data can be grouped into the following categories: building detection, building roof contour extraction, building roof extraction, and building model extraction. Building detection is performed using a digital surface model (DSM) [9], a normalized DSM (nDSM) [10], or an ALS point cloud [11]. Building roof boundary extraction usually involves the detection of irregular roof boundaries, 
which are further subjected to a regularization process [12][14]. Building roof extraction uses segmentation methods to group ALS point cloud data into planar faces and requires a homogeneity criterion, such as approximate height similarity and/or approximate normal vector similarity [15], [16]. Building model extraction usually involves building detection, roof contour extraction, and roof extraction [17], [18].

As was discussed above, methods that are based on the combination of photogrammetric and ALS data seek to take advantage of the synergy between both data sources. ALS data are superior in terms of height accuracy, and image data are superior in terms of boundary definition. Thus, many extraction schemes that involve both image and ALS data have been developed to improve the quality of building roof boundaries. Haala and Brenner [19] combined multispectral imagery and ALS-derived DSM to separate buildings from vegetation. In Sohn and Dowman [20], buildings were first extracted from Ikonos imagery and an ALS-derived DSM, and the results from both data sources were combined to remove inconsistencies. Vosselman [21] combined the ALS point cloud, plan view, and high-resolution aerial image data to automatically reconstruct 3-D buildings. In [22] and [23], buildings were segmented in ALS data and refined using an aerial image. Kim and Habib [24] proposed a method for the automated generation of polyhedral building models for complex structures that starts with the extraction of individual planar patches from the ALS point cloud, refines them with image data, and generates the polyhedral building model with the refined building patches. Cheng and Zhao [25] proposed an approach that integrates QuickBird imagery and ALS-derived nDSM to detect buildings in which the nDSM is used to eliminate ground points, and the image is used to detect buildings. In [26], isolated roof planes segmented in an ALS point cloud and straight lines extracted from a high-resolution image are integrated into a consistent structure, i.e., the 3-D roof model. Aiming at reconstructing digital building models, Zhou and Zhou [27] proposes a seamless fusion between the LiDAR and aerial imagery on the basis of aspect graphs, which utilize the features of houses, such as geometry, structures, and shapes. In Zarea and Mohammadzadeh [28], an nDSM (generated by preprocessing an ALS point cloud) and orthorectified aerial image is used in a support vector machine classification procedure for separating building and trees and, in addition, resulting building regions are postprocessed by the $k$-means cluster algorithm to separate buildings with different heights. Other recent papers (e.g., [1], [8], [29]) have reported methods that integrate both data sources to detect or delineate building roof boundaries.

This paper proposes a method for extracting groups of straight lines that represent roof boundary sides and roof ridgelines from high-resolution aerial images using corresponding ALS-derived roof polyhedrons as initial approximations. This research is motivated by the possibility of using straight lines in the image space that represent building roof details in several applications. For example, straight lines that represent roof boundary sides can be back-projected onto the ALS-derived building polyhedron to refine the accuracy of its boundary. Moreover, roof straight lines can be used to refine the exterior orientation parameters of unmanned aerial vehi-

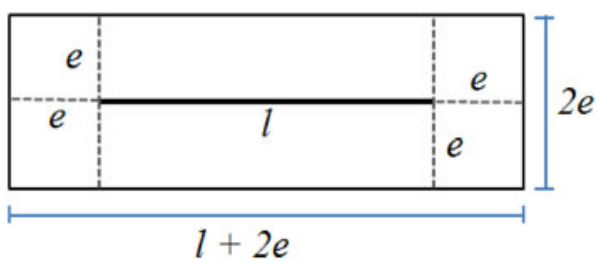

Fig. 1. Bounding box.

cle (UAV) images assuming that they are sometimes not accurately determined during the flight mission using the integrated GPS-inertial systems. The remainder of this paper is organized as follows. Section II describes the proposed method, the results are presented and discussed in Section III, and Section IV presents the main conclusions.

\section{Proposed Method for Extraction of Straight LINE GROUPS}

The proposed method is based on two main steps. First, straight lines that are candidates to represent roof ridgelines and roof boundary sides of a building are extracted from the aerial image. A group of straight lines that represent roof boundary sides and ridgelines of a selected building is then obtained through the optimization of an MRF-based energy function using the genetic algorithm (GA) optimization method.

\section{A. Extraction of Straight Lines}

The input data include ALS-derived roof polyhedrons and an aerial image (along with orientation parameters). The first step consists of transforming the vertices of a roof polyhedron into the image space. Mathematically, this requires standard photogrammetric treatments using the camera model. Bounding boxes are then generated around the projected straight lines, which are defined by two adjacent projected roof polyhedron vertices (see Fig. 1) as follows:

1) each projected straight line coincides with the axis of symmetry of the corresponding bounding boxes, and

2) the dimensions of each bounding box are calculated based on the length $(l)$ of the corresponding projected straight line and the maximum projection error $(e)$ of the endpoints.

The error propagation law [30] is the basis for estimating the projection error in the transformation of a point in object space into the corresponding point in the image space. The length and width of each bounding box are $l+2 e$ and $2 e$, respectively.

The next step consists of applying the Canny edge detector [31] and the Steger line detector [32] within each bounding box followed by an edge-linking algorithm [33] and the DouglasPeucker polygonization algorithm [34]. The application of the Canny and Steger detectors within the bounding boxes instead of applying them to the entire image is intended to avoid the extraction of straight lines that have no chance of representing a boundary side or ridgeline of a roof. The rationality of combining both detectors is that roof boundaries and ridgelines in very-high-resolution aerial images exhibit different image profiles (see Fig. 2). Most roof boundaries have step-edge profiles, while most roof ridgelines have line profiles. Fig. 2(a) shows 


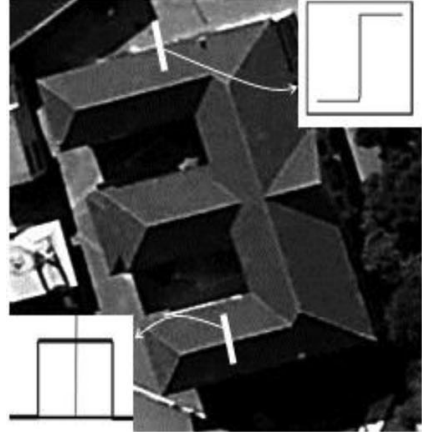

(a)

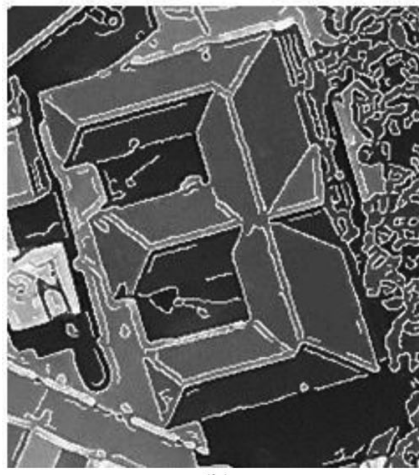

(b)

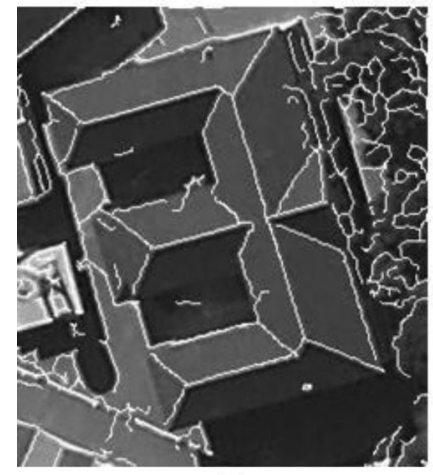

(c)

Fig. 2. Lines and edges. (a) Step-edge model (top-right corner) and Line model (bottom-left corner). (b) Edges detected by the Canny detector. (c) Lines detected by the Steger detector.

(top-right corner of the image) the step-edge model for profiles taken at roof boundary points and the line model (or bar-shaped model [31] - bottom-left corner of the image) for profiles taken at roof ridgeline points. This means that the Canny edge detector usually detects only one edge per roof boundary and two near and parallel edges per roof ridgeline [see Fig. 2(b)]. Conversely, the Steger detector usually detects only one line per roof ridgeline [see Fig. 2(c)]. Moreover, ridgelines that are detected by the Steger detector approximately coincide with the axes of symmetry of the corresponding double edges that are detected by the Canny detector.

As a result, the following basic algorithm for extracting straight lines that represent roof boundary sides and ridgelines with a minimum number of false positives is proposed:

1) apply the Canny and Steger detectors within all of the bounding boxes;

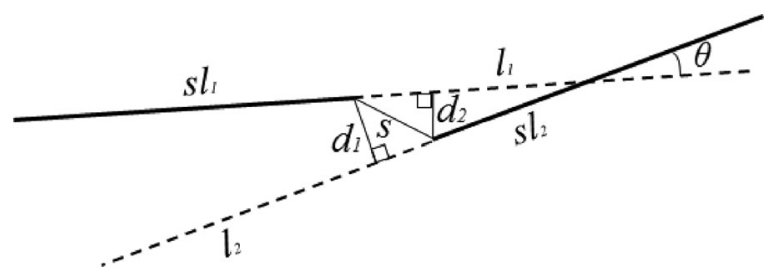

Fig. 3. Collinearity and proximity condition.

2) organize the edge points into straight lines using the edgelinking and Douglas-Peucker algorithms; and

3) check the pairs of Canny-derived straight lines for parallelism and proximity and eliminate the pairs that match this criterion and that have a Steger-derived straight line between them. The remaining straight lines within all of the bounding boxes are candidates to represent the corresponding roof boundary sides or ridgelines.

To filter the extracted straight-line segments that have very low chances of representing roof boundary sides or ridgelines, those that are too short are discarded. Moreover, straight lines that form large angles with the corresponding projected straight line are also discarded. Short straight-line segments usually come from noisy images. On the other hand, the filtering with the orientation criterion is intended to remove straight-line segments that result from adjacent roof edges or ridgelines that are within the current bounding box. These straight-line segments are usually very short, but when the width of the bounding box is relatively large, the orientation criterion is necessary to filter these undesired straight-line segments.

Finally, the straight-line segments are postprocessed to reduce the fragmentation of the edge or line detection results. Two or more straight-line segments are merged if they comply with the continuity rule (see Fig. 3): they should be collinear, which means that the angle $(\theta)$ between them should be less than a predefined threshold; the transversal separation $(d)$ between them should be less than a predefined threshold, and any of their endpoints cannot be projected onto each other; and they should be sufficiently close, which means that the distance $(s)$ between the closest endpoints should be less than a predefined threshold. The parameter $d$ is defined by averaging the distances $d_{1}$ and $d_{2}$ (i.e., $d=0.5\left(d_{1}+d_{2}\right)$ ), where $d_{1}$ is the distance between the endpoint of the straight line segment $s l_{1}$, which is closest to the endpoint of the adjacent straight line segment $\left(s l_{2}\right)$, and the support line $\left(l_{2}\right)$ of the straight line segment $s l_{2}$, and the distance $d_{2}$ is defined similarly. Because $\theta$ and $s$ are assumed to be small, $d \approx d_{1} \approx d_{2}$.

This algorithm allows for the extraction of $n$ sets of straight lines, each of which is a candidate to represent a roof boundary side or ridgeline.

\section{B. Identification of Straight Lines That Represent Roof Ridgelines and Roof Boundary Sides Using an MRF Model}

1) Basics of the MRF Theory: The MRF model has the advantage of characterizing the contextual knowledge by modeling the spatial relationships between the primitives (e.g., segments of lines, contours) that represent real-world objects. In an MRF, 
the primitives $R_{1}, R_{2}, \ldots, \mathrm{R}_{\mathrm{n}}$ (where $R$ is the set of primitives; i.e., $R=R_{1}, R_{2}, \ldots, R_{n}$ ) are related to one another according to a neighboring system $N=\left\{N\left(R_{1}\right), N\left(R_{2}\right), \ldots, N\left(R_{n}\right)\right\}$, where $N\left(R_{i}\right), i=1,2, \ldots, n$, is the set of all of the primitives in $R$ in the neighborhood of $R_{\mathrm{i}}$.

Let $X=X_{1}, X_{2}, \ldots, X_{n}$ be a family of random variables that are defined over $R$, where each $X_{i}$ corresponds to $R_{i}$. The $n$-dimensional random variable $X$ is an MRF over $R$ with respect to the neighborhood system $N$ if $P[\mathrm{X}=\mathrm{x}]>0$ for all $X$ realizations, and $P\left[X_{i}=x_{i} \mid X_{j}=x_{j} \forall j \neq i\right]=P\left[X_{i}=x_{i} \mid X_{j}=\right.$ $\left.x_{j} \forall j \mid R_{j} \in N\left(R_{i}\right)\right]$.

According to the Hammersley-Clifford theorem [35], an MRF can also be characterized by a Gibbs distribution, i.e.,

$$
P[X=x]=\frac{1}{Z} \exp ^{-U(x)}
$$

where $x$ is a realization of $X, Z$ is a normalization constant, and $U(x)$ is the Gibbs energy function

$$
U(x)=\sum_{c \in C(R, N)} V_{c}(x) .
$$

Equation 2 shows that the energy function is the sum of the clique potentials $(\mathrm{Vc}(\mathrm{x}))$ over all possible cliques $c \in C(R, N)$. A clique $c$ is a subset of the primitives in $R$, such that every primitive neighbors one another. The set of all of the cliques over $R$ with respect to the neighborhood system $N$ is represented by $C(R, N)$. The value of $V c(\mathrm{x})$ depends on the local configuration of the clique $c$.

2) MRF-Based Energy Function: The straight lines that are extracted from the image around the edges of the projected roof polyhedron are used to build an MRF model that expresses specific forms of building roofs with reference to the projected roof polyhedron. The energy function is defined so that each straight line is associated with a random variable $\left(x_{\mathrm{i}}\right)$, which takes binary values according to the following rule:

$$
x_{i}=\left\{\begin{array}{cc}
1 & \text { iff the } i \text { th }- \text { straight line is true positive } \\
0 & \text { otherwise }
\end{array}\right.
$$

This rule (3) results in an $n$-dimensional random vector, where $n$ is the number of straight lines. This random vector is unknown that is determined with an optimization process.

Fig. 4 shows a hypothetical example of a building roof that is projected onto the image space. The projected straight lines are $f_{1}, \ldots, f_{n}$. We assume that $\mathrm{n}_{\mathrm{k}}$ straight lines were extracted around the projected straight line $f_{k}$. Fig. 4 shows only the $i$ th straight line $\left(\mathrm{F}_{\mathrm{i}}\right)$ that was extracted from the $f_{\mathrm{k}}$ bounding box, the $l$ th straight line $\left(\mathrm{F}_{1}\right)$ that was extracted from the $f_{k+1}$ bounding box, and the $j$ th straight line $\left(\mathrm{F}_{\mathrm{j}}\right)$ that was extracted from the $f_{k+2}$ bounding box. We say that the projected straight line $f_{k}$ (or either $f_{k+1}$ or $f_{k+2}$ ) is the nearest line to the extracted straight line $F_{i}$ (or either $F_{l}$ or $F_{j}$ ). $q_{i l}$ is a corner detected by a corner detection algorithm around the intersection between the extracted straight lines $F_{i}$ and $F_{l}$. These elements, and others that are explained just below, are sufficient to understand the formulations of all energy terms.

The energy function is composed of five energy terms as follows:

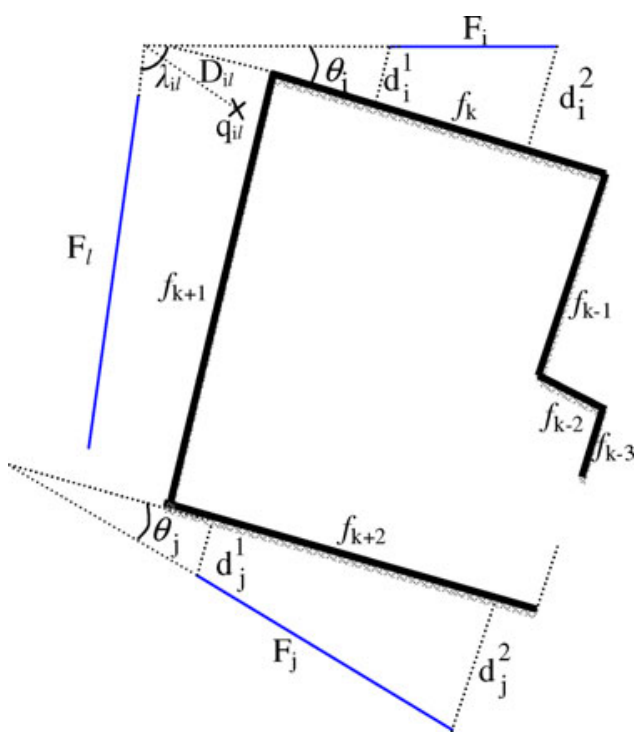

Fig. 4. Geometric elements for defining the energy terms.

1) The first term (4) favors long straight lines with reference to the nearest projected straight line. It is called the relative length energy term

$$
U_{1}(x)=\sum_{i=1}^{n} x_{i} \frac{L_{f_{k}}}{L_{F_{i}}}
$$

where $L_{f_{k}}$ is the length of the projected straight line $\left(f_{\mathrm{k}}\right)$ that is nearest the $i$ th straight line that was extracted from the image $\left(F_{i}\right)$, and $L_{F_{i}}$ is the length of the extracted straight line $\left(\mathrm{F}_{\mathrm{i}}\right)$ (see Fig. 4).

2) The second term of the energy function (5) is called the proximity term and is defined for two extracted straight lines (e.g., $F_{i}$ and $F_{j}$ ) that are in the neighborhood of two different projected straight lines ( $f_{k}$ and $f_{k+2}$ considering $F_{i}$ and $F_{j}$, respectively). This term favors a pair of straight lines that is closer to the projected straight lines

$$
U_{2}(x)=\sum_{i=1}^{n} \sum_{j \mid j \in N_{i}} x_{i} \cdot x_{j} \cdot P(i, j)
$$

where $P(i, j)=\frac{1}{2}\left(d_{i}^{1}+d_{i}^{2}+d_{j}^{1}+d_{j}^{2}\right), d_{i}^{1}$ and $d_{i}^{2}$ are the distances between the endpoints of the extracted straight line $F_{\mathrm{i}}$ and the nearest projected straight line $\left(\mathrm{f}_{\mathrm{k}}\right)$, and $d_{j}^{1}$ and $d_{j}^{2}$ are the distances between the endpoints of the extracted straight line $F_{\mathrm{j}}$ and the nearest projected straight line $\left(\mathrm{f}_{\mathrm{k}+2}\right)$.

3) The third term is called the orientation term (6) and favors a pair of extracted straight lines (e.g., $F_{i}$ and $F_{j}$ ) with similar orientations to the nearest projected straight lines ( $f_{k}$ and $f_{k+2}$ considering $F_{i}$ and $F_{j}$, respectively)

$$
U_{3}(x)=\sum_{i=1}^{n} \sum_{j \mid j \in N_{i}} x_{i} . x_{j} . s_{\theta}(i, j)
$$


where $s_{\theta}=\frac{2}{1+\exp \left[-\beta \cdot\left(\theta-\theta_{0}\right)^{2}\right]}-1, \theta=\theta_{i}+\theta_{j}, \theta_{i}$ is the angle between the extracted straight line $F_{i}$ and the nearest projected straight $\left(\mathrm{f}_{\mathrm{k}}\right), \theta_{j}$ is the angle between the extracted straight line $F_{\mathrm{j}}$ and the nearest projected straight line $\left(\mathrm{f}_{\mathrm{k}+2}\right), \beta$ is a positive constant, and $\theta_{0}$ is the optimal value $\left(0^{\circ}\right.$ or $\left.180^{\circ}\right)$ of the parameter $\theta$.

4) The fourth term is called the rectangularity term and favors configurations of extracted straight lines that intersect at approximately right angles. This occurs with rectilinear roof boundaries. This property is usually not valid for straight lines that represent roof ridgelines. The rectangularity term is as follows:

$$
U_{4}(x)=\sum_{i=1}^{n} \sum_{j \mid j \in N_{i}} x_{i} \cdot x_{j} \cdot\left|\operatorname{sen}\left(2 \alpha_{i j}\right)\right|
$$

where $\alpha_{i j}$ is the angle between the extracted straight lines $F_{i}$ and $F_{j}$, each of which neighbors two adjacent projected straight lines.

5) The fifth term is called the corner term and should favor extracted straight lines that intersect near a corner in the image where two roof edges intersect each other at approximately a right angle. This principle can be mathematically represented as

$$
U_{5}(x)=\sum_{i=1}^{n} \sum_{j \mid j \in N_{i}} x_{i} \cdot x_{j} \cdot D_{i j} \cdot \cos \left(\lambda_{i j}\right)
$$

where $D_{i j}$ is the Euclidian distance between the intersection point of two extracted straight lines $\left(F_{i}\right.$ and $\left.F_{j}\right)$ that neighbor two adjacent projected straight lines and the nearest corner $\left(q_{\mathrm{ij}}\right)$ detected in the image, and is the angle between the edges that define the corner $q_{i j}$.

It is worth noting that the fourth and fifth energy terms should only be applied for straight line candidates to represent roof boundary sides. Roof ridgelines usually do not intersect at right angles. Finally, the energy equation is formulated as follows:

$$
U(x)=k_{1} U_{1}(x)+\ldots+k_{5} U_{5}(x)
$$

where $k_{1}, \ldots, k_{5}$ are positive constants $\sum_{1}^{5} k_{i}=1$.

\section{Optimization of the Energy Function}

The optimal configuration $x$ is obtained by minimizing the energy function $U(x)$, i.e., $x=\operatorname{argmin}(U(x))$. We employed the GA.

GAs are based on evolutionary ideas of natural selection. They exploit a random search to solve optimization problems. The search space comprises a population of individuals (chromosomes) that represent possible solutions to a problem. A random vector of components represents each individual. Usually, each component is coded to a binary value (i.e., 0 or 1 ). After randomly selecting an initial population, the algorithm proceeds by employing the following three operators:

1) selection, which equates to survival of the fittest;

2) crossover, which represents the coupling between individuals; and

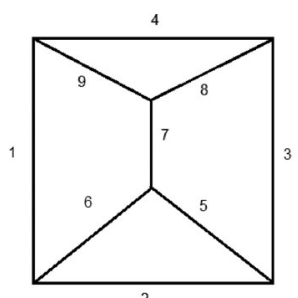

(a)

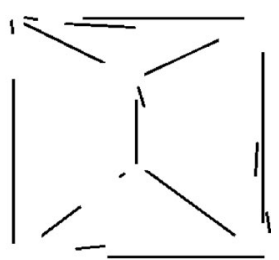

(b)
Fig. 5. Illustrative example. (a) Projected ALS-derived roof polyhedron; (b) extracted straight lines.

3) mutation, which introduces random modifications.

The GA optimization converges when the fittest individual is good enough according to a fitness measurement. For more details, please refer to the relevant literature, such as [36].

To initialize the optimization process by the GA, an initial population should be established. The initial population should be established by considering that each roof side or ridgeline has only one corresponding extracted straight line. We refer to this as the uniqueness heuristic. Each individual is generated by randomly selecting a candidate (extracted) straight line that may represent a roof boundary side or roof ridgeline.

Fig. 5 illustrates the generation of the initial example. Fig. 5(a) shows the projected ALS-derived roof polyhedron, and Fig. 5(b) shows the extracted straight lines around the roof boundary and ridgelines. It is worth noting that only one or very few candidates represent the projected roof ridgelines. This is expected because the combination of the Canny edge detector and the Steger line detector usually provides a unique response for each roof ridgeline. On the other hand, the preprocessing step usually selects two or more candidates to represent each roof boundary side.

Fig. 6 shows an example of an initial population of $n$ individuals that is generated by taking into account the uniqueness heuristic. Each individual is generated by randomly selecting a candidate straight line to represent each roof boundary side or ridgeline. Fig. 6 is organized as follows. The first line identifies the roof boundary sides $(1, \ldots, 4)$ and the roof ridgelines $(5$, $\ldots, 9)$, and the second line identifies the candidate (extracted) straight lines $(1, \ldots, 17)$. The other lines show the individuals that make up the initial population. A 17-D binary vector represents each individual. In other words, 17 genes form an individual. When a straight line is selected to represent a roof boundary side or a roof ridgeline, it is set to 1 (one); otherwise, it is set to 0 (zero). For example, the first individual shows that the straight lines $1,4,6$, and 8 and the lines $11,12,15,16$, and 17 are candidates to represent the roof boundary sides $1, \ldots, 4$ and the roof ridgelines $5, \ldots 9$, respectively.

Each individual (i.e., a straight-line configuration that represents the roof boundary sides and ridgelines) of the initial population is a possible solution to the optimization problem; it is a point in the solution space. After the initial population is generated, the fitness of every individual is computed using the fitness function (i.e., the energy function that is given by (9)), 


\begin{tabular}{|c|c|c|c|c|c|c|c|c|c|c|c|c|c|c|c|c|c|}
\hline & \multicolumn{2}{|c|}{1} & \multicolumn{2}{|c|}{2} & \multicolumn{3}{|c|}{3} & \multicolumn{3}{|c|}{4} & 5 & \multicolumn{2}{|c|}{6} & \multicolumn{2}{|c|}{7} & 8 & 9 \\
\hline Candidate straight lines & 1 & 2 & 3 & 4 & 5 & 6 & 7 & 8 & 9 & 10 & 11 & 12 & 13 & 14 & 15 & 16 & 17 \\
\hline Individuals & & & & & & & & & & & & & & & & & \\
\hline 1 & 1 & 0 & 0 & 1 & 0 & 1 & 0 & 1 & 0 & 0 & 1 & 1 & 0 & 0 & 1 & 1 & 1 \\
\hline 2 & 1 & 0 & 1 & 0 & 1 & 0 & 0 & 1 & 0 & 0 & 1 & 1 & 0 & 1 & 0 & 1 & 1 \\
\hline 3 & 0 & 1 & 1 & 0 & 0 & 0 & 1 & 0 & 1 & 0 & 1 & 0 & 1 & 1 & 0 & 1 & 1 \\
\hline $\mathbf{n}$ & 0 & 1 & 0 & 1 & 0 & 0 & 1 & 0 & 0 & 1 & 1 & 0 & 1 & 0 & 1 & 1 & 1 \\
\hline
\end{tabular}

Fig. 6. Initial population.

\begin{tabular}{|c|c|c|c|c|c|c|c|c|c|c|c|c|c|c|c|c|c|}
\hline & \multicolumn{2}{|c|}{1} & \multicolumn{2}{|c|}{2} & \multicolumn{3}{|c|}{3} & \multicolumn{3}{|c|}{4} & 5 & \multicolumn{2}{|c|}{6} & \multicolumn{2}{|c|}{7} & \multirow{2}{*}{$\begin{array}{c}8 \\
16 \\
\end{array}$} & \multirow{2}{*}{$\begin{array}{c}9 \\
17 \\
\end{array}$} \\
\hline & 1 & 2 & 3 & 4 & 5 & 6 & 7 & 8 & 9 & 10 & 11 & 12 & 13 & 14 & 15 & & \\
\hline \multicolumn{18}{|c|}{ Pair of individuals } \\
\hline 1 & 1 & 0 & 0 & 1 & 0 & 1 & 0 & 1 & 0 & 0 & 1 & 1 & 0 & 0 & 1 & 1 & 1 \\
\hline 2 & 1 & 0 & 1 & 0 & 1 & 0 & 0 & 1 & 0 & 0 & 1 & 1 & 0 & 1 & 0 & 1 & 1 \\
\hline \multicolumn{18}{|c|}{ Crossover } \\
\hline 1 ' & 1 & 0 & 0 & 1 & 0 & 1 & 0 & 1 & 0 & 0 & 1 & 1 & 0 & 1 & 0 & 1 & 1 \\
\hline 2' & 1 & 0 & 1 & 0 & 1 & 0 & 0 & 1 & 0 & 0 & 1 & 1 & 0 & 0 & 1 & 1 & 1 \\
\hline
\end{tabular}

Fig. 7. Crossover

\begin{tabular}{|c|c|c|c|c|c|c|c|c|c|c|c|c|c|c|c|c|c|}
\hline & \multicolumn{2}{|c|}{1} & \multicolumn{2}{|c|}{2} & \multicolumn{3}{|c|}{3} & \multicolumn{3}{|c|}{4} & 5 & \multicolumn{2}{|c|}{6} & \multicolumn{2}{|c|}{7} & \multirow{2}{*}{$\begin{array}{c}8 \\
16 \\
\end{array}$} & \multirow{2}{*}{$\begin{array}{c}9 \\
17 \\
\end{array}$} \\
\hline & 1 & 2 & 3 & 4 & 5 & 6 & 7 & 8 & 9 & 10 & 11 & 12 & 13 & & 15 & & \\
\hline Individual & & & & & & & & & & & & & & & & & \\
\hline 1 & 1 & 0 & 0 & 1 & 0 & 1 & 0 & 1 & 0 & 0 & 1 & 1 & 0 & 1 & 0 & 1 & 1 \\
\hline Mutation & & & & & & & & & & & & & & & & & \\
\hline 1' & 1 & 0 & 1 & 0 & 0 & 1 & 0 & 1 & 0 & 0 & 1 & 1 & 0 & 1 & 0 & 1 & 1 \\
\hline
\end{tabular}

Fig. 8. Mutation.

which allows the selection of the fittest individual for the current iteration. A new population is then generated from the current fittest individual using the selection, crossover, and mutation genetic operators.

The selection of individuals is based on the return of the fitness function. In this paper, we use selection by deterministic sampling [36] because poorer individuals may have some features that should be retained and used in the following steps. Moreover, it is possible that the fittest individuals have no such features, and the selection of only these individuals can result in a local convergence. The selected reproduction technique is the exchange of the entire population by elitism [36]. The fittest individual is copied into the following population, and $n$ - 1 individuals are replaced using the genetic operators (i.e., the crossover and mutation operators). Details of these genetic operations are discussed below.

The crossover operation consists of randomly forming pairs of individuals with a given probability of swapping genes between two individuals from a cut point (see Fig. 7). Considering the uniqueness of heuristics, the random cut-off value must correspond to one roof boundary side or roof ridgeline. In Fig. 7, the arrow corresponds to the cut point, which is the fourth roof boundary side.

The crossover operation generates two new individuals; one has the genes of the first old individual from the cut point backward and the genes of the second old individual from the cut point forward; and the second has the opposite genes. Because the crossover has a certain probability of occurrence, when it does not take place, the old individuals remain in the new population.

After the crossover, there is a probability of mutation in a certain individual. When a mutation occurs, the uniqueness heuristic must be considered when selecting the mutation point (cut point; the arrow in Fig. 8). We apply the mutation by inversion (see Fig. 8). The probability of mutation is usually set to a low value. 
After creating the new population, the fitness of every individual is calculated to determine the new fittest individual. The optimization process is iterated until it reaches a predetermined stop criterion. In this study, a maximum number of generations (i.e., iterations) is defined. The result of the GA optimization process is the individual that ultimately has the highest fitness. The fittest individual is the best configuration of the selected straight lines to represent the roof boundary sides and roof ridgelines.

This model has two drawbacks as follows:

1) For straight lines that are candidates to represent a roof boundary side or a roof ridgeline, one is assumed as correct; and

2) some roof boundary sides or ridgelines may not have any candidate, which requires that the corresponding projected straight lines should be assumed to be correct.

\section{EXPERIMENTAL RESULTS}

\section{A. Dataset and Parameter Setting}

The dataset that was used in our three first experiments includes ALS-derived polyhedrons that represent building roofs, which were extracted from an ALS point cloud with a density of approximately 2 points $/ \mathrm{m}^{2}$, and 20-cm digital aerial images along with interior and exterior orientation parameters. The fourth experiment used a $6 \mathrm{~cm}$ digital aerial image and roof polyhedrons derived from an 8 points $/ \mathrm{m}^{2}$ ALS point cloud.

The following parameters and thresholds were used:

1) Altimetric and planimetric precisions of the ALS points: 15 and $48 \mathrm{~cm}$, respectively;

2) Thresholds for analyzing the continuity between two straight lines: $\theta=9^{\circ}, s=4$ pixels, and $d=2$ pixels;

3) Constant that controls the shape of the sigmoid function: $\beta=20$;

4) Weights that control the relative importance of the energy terms of the energy function: $k_{2}=0.1 ; k_{4}=0.3 ; k_{1}=$ $k_{3}=k_{5}=0.2$;

5) Crossover and mutation probabilities: 0.7 and 0.01 , respectively;

6) Threshold for filtering noisy straight lines using the length criterion: $0.15 l$, where $l$ is the length of the projected straight line that is nearest to the extracted straight line to be tested;

7) Threshold for filtering straight lines using the orientation criterion: $9^{\circ}$.

All these parameters and thresholds were kept constant in all of the experiments. However, the size of the initial population ( $n$ ) and the maximum number of generations (or iterations) require different values because both parameters depend on the complexity of the roof; these parameters were set to 15 and 20 , respectively, for the buildings of the two first experiments and for the buildings A, C, D, E, and F of the fourth experiment, and to 25 and 40 , respectively, for the building of the third experiment and for the building B of the fourth experiment. Other exceptions involve the Canny and Steger detectors, which require appropriate values for the Gaussian scale parameter $(g s)$ and the low $\left(h_{1}\right)$ and high $\left(h_{h}\right)$ hysteresis parameters. Because

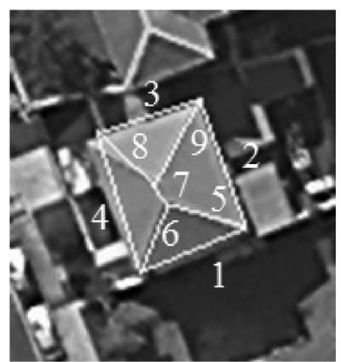

(a)

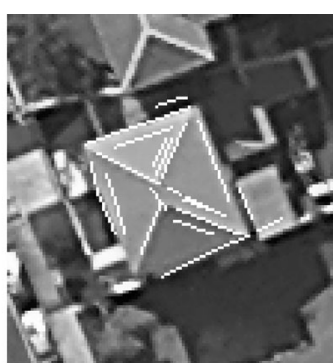

(b)

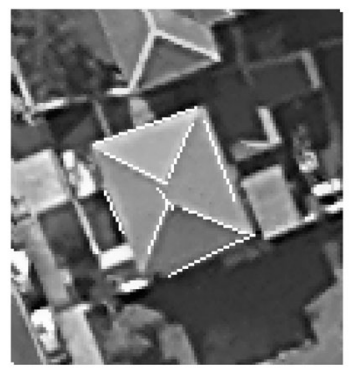

(c)

Fig. 9. Test 1. (a) Projected ALS-derived roof polyhedron; (b) straight lines extracted from the image; and (c) group of straight lines obtained by the GA optimization.

these three parameters depend on the signal-to-noise ratio of each image, different setups are expected for different images. In this case, the first three experiments use the same image, so the Canny parameters are $g s=1.8, h_{l}=0.8$, and $h_{h}=5.0$, and the Steger parameters are $g s=1.2, h_{l}=0.5$, and $h_{h}=5.0$. On the other hand, for the fourth experiment, the Canny parameters are $g s=1.5, h_{l}=3$, and $h_{h}=20$ and Steger parameters are $g s=1.2, h_{l}=3.0$, and $h_{h}=8.0$.

\section{B. Experiments and Discussion}

To evaluate the proposed method, several experiments were accomplished by using real data. The results obtained will be analyzed visually and numerically. The numerical analysis will be based on the following quality parameters: the number of false negatives and false positives; the accuracy index (AI) of each extracted straight line, defined as the difference between 1) the average displacement between a projected ALS-derived straight line and the corresponding roof image edge (rie), and 2) the average displacement between the extracted straight line and the same rie; and the completion index (CI) of each extracted straight line, which is defined as the ratio (in \%) between the length of the extracted straight line and the length of the corresponding rie. Four experiments are presented and discussed in the following.

Fig. 9 (Test 1) shows a building roof that is defined by four sides and five ridgelines. Fig. 9(a) shows the projected ALSderived roof polyhedron overlaid on the image. Note that the projected straight lines 3,4 , and 8 coarsely match the corresponding roof boundary sides or roof ridgelines. Moreover, registration errors are visible along the other roof boundary sides and ridgelines.

Fig. 9(b) shows 19 straight lines that were extracted using the preprocessing techniques that were proposed in Section II-A. 
They are candidates to represent roof boundary sides and roof ridgelines. Roof ridgelines 6 and 8 each have only one candidate, roof boundary sides 1 and 2 each have two candidates, roof boundary sides 3 and 4 and roof ridgelines 5 and 9 each have three candidates, and ridgeline 7 has two candidates. Moreover, the roof boundary side 4 has two candidate straight lines that are too near and collinear and roof boundary sides 1,2 , and 3 have candidates that belong to the ground or to a neighboring building's roof. The larger the registration error, the larger the area within the bounding box and, as a result, the higher the probability of extracting straight lines from adjacent objects. Fig. 9(c) shows the result of the optimization process. Although most of the candidate sets involve two or more nearly parallel straight lines, the optimization process was able to find all of the correct correspondences. Moreover, roof boundary sides 1 and 2 have candidate straight lines that belong to a neighboring building. In all of these cases, the correct candidates are the longest ones and are also nearer to the corresponding projected straight lines, which favor the minimization of the relative length and proximity energy terms. The extraction of the correct corner also helps in the global minimization of the energy function. For example, the two corrected candidate straight lines that were extracted from around projected straight lines 2 and 3 have the highest probability of intercepting near a right-angle roof corner; in this example, this is the image corner that is defined by roof edges 2 and 3. In this test, no false positive or negative is verified and the accuracy of the grouping of extracted straight lines has been improved. The average improvement in accuracy (i.e., the average $\mathrm{AI}$ ) is around 2 pixels and the maximum improvement in accuracy (maximum AI) reaches 4 pixels for the roof ridgeline 8. The CI has also been computed for all extracted straight lines. The best result is found for the roof boundary side $3(\mathrm{CI}=100 \%)$ and the worse one for the roof boundary side $4(\mathrm{CI}=40 \%)$. The average $\mathrm{CI}$ is around $85 \%$, indicating that the proposed approach had a satisfactory completion ratio for this test.

Fig. 10 shows the second test building. Fig. 10(a) shows the projected ALS-derived roof polyhedron superposed on the image. It is worth noting that a large registration error is clearly observed because the projected ALS-derived roof polyhedron is displaced to the right. Eighteen straight lines were extracted around the roof boundary sides and ridgelines [see Fig. 10(b)].

As in the previous example, the preprocessing step retains few candidates that represent every roof boundary side and ridgeline. All of the boundary sides have two candidates, four ridgelines have one or two candidates, and one ridgeline has four candidates. However, few straight lines are extracted from adjacent objects due to the large registration error. Fig. 10(c) shows the result of optimizing the MRF-based energy function using the GA. The method correctly matches the candidate straight lines to the corresponding projected ALS-derived straight lines; in other words, no false negatives or positives occur in this example. Also, the accuracy is better for all extracted straight lines, i.e., the AI ranges from 2 (obtained for the roof boundary sides 1 and 3) to 12 pixels (obtained for the roof boundary sides 2,4 , and 7). This relatively high improvement in accuracy is directly related to the large registration error of the projected ALS-derived roof polyhedron [see Fig. 10(a)]. Due to this, the

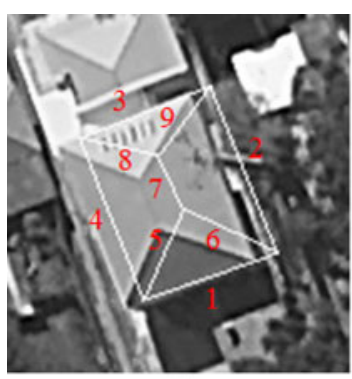

(a)

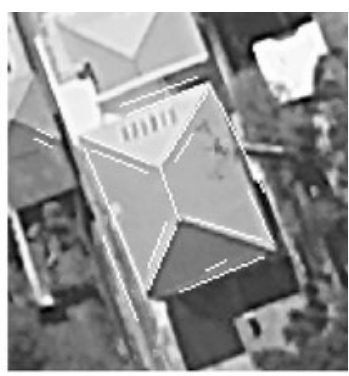

(b)

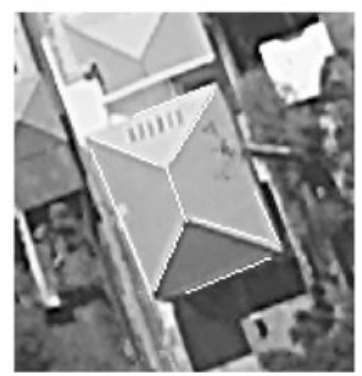

(c)

Fig. 10. Test 2. (a) Projected roof; (b) extracted lines of the image; and (c) results of the energy function optimization.

average improvement in accuracy (AI) is around 7 pixels. In general, the extraction method reaches a good completion ratio $(\mathrm{CI}=82 \%$ in average), getting the worse result for the roof boundary side $2(\mathrm{CI}=30 \%)$ and the best one $(\mathrm{CI}=100 \%)$ for the roof ridgeline 5 .

Fig. 11 shows the third test building, which is much more complex than those in the first two experiments. Nineteen roof boundary sides and 21 roof ridgelines form the roof structure of this building. It is composed of a main roof branch, which is connected orthogonally to three secondary roof branches; it is a composite of four hipped roofs. Fig. 11(a) shows the projected ALS-based roof polyhedron overlaid on the image. In general, the projected polyhedron edges are relatively near the corresponding roof boundary sides and ridgelines, but registration errors are visible along many of them. The projected straight lines 13 and 15 are coarse representations of details around the end of the central secondary roof branch. Fine details are visible in this area, which are difficult to model using the available ALS point cloud mainly due to its low point density. Fig. 11(b) shows the straight lines that were extracted from the image using the techniques that were described in Section II-A. Most of the roof boundary sides and ridgelines have only one candidate to represent them. The main reason for this is the high quality of the interior and exterior orientation parameters that were used to project the ALS-based building polyhedron edges because of the low registration error. As a result, the area within the bounding boxes is small, which allows the extraction of few candidate straight lines. The registration quality also depends on the quality of the ALS-based roof polyhedron. Fig. 11(b) also shows that one roof boundary side (7) and three ridgelines $(33,34$, and 36 ) have two candidates and that four roof boundary sides $(11,13,15$, and 17) have no candidate. Whenever a roof boundary side or a roof 


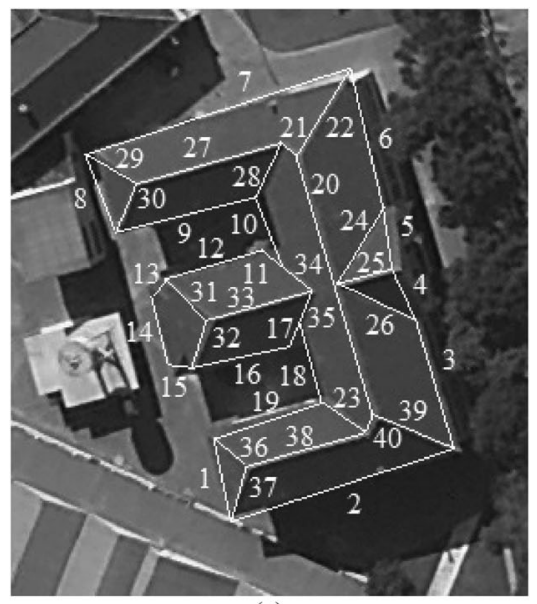

(a)

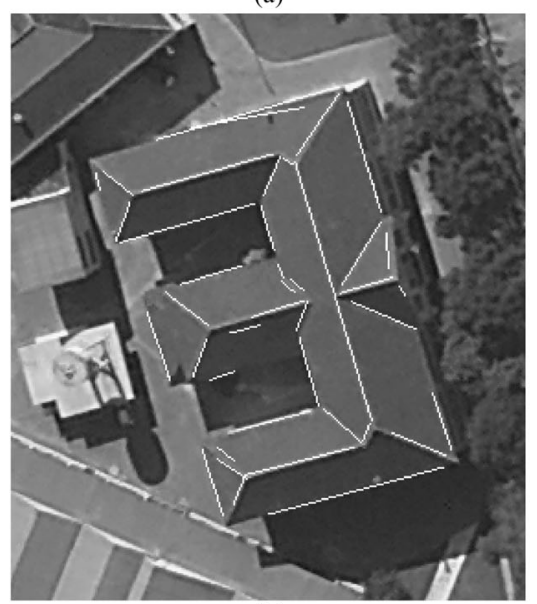

(b)

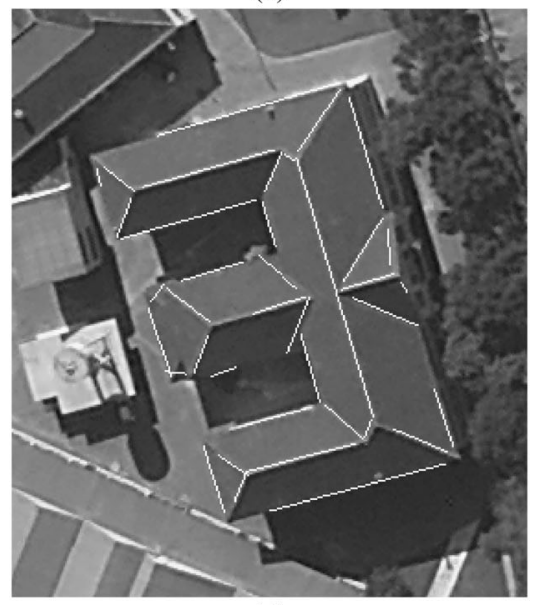

(c)

Fig. 11. Test 3. (a) Projected roof; (b) extracted lines of the image; and (c) results of the energy function optimization.

ridgeline does not have a candidate, the corresponding projected straight line is assumed to be the candidate. This is helpful in establishing all possible spatial relationships between the extracted straight lines, which is fundamental to constructing a robust MRF-based energy function. The results that were obtained by optimizing the proposed energy function using the GA are shown in Fig. 11(c). The method successfully identified $36(90 \%)$ straight lines. The method failed in finding four (false negatives) correspondences, even though these failures were expected because the preprocessing approach was not able to provide candidates for roof boundary sides 11, 13, 15, and 17 . As in previous tests, no false positive is verified. Most of the projected ALS-derived straight lines are correctly positioned in the image and the same occurs for the corresponding extracted straight lines, mainly those that represent roof ridgelines. However, more significant accuracy improvements (AI of about 4 pixels) are observed for the roof boundary sides 8 and 10 . The average AI is about 2 pixels, showing a relatively low registration error of the projected ALS-derived roof polyhedron. The CI for almost one-third of the extracted straight lines reaches the optimal value, i.e., $\mathrm{CI}=100 \%$. On the other hand, the worse CI of about $35 \%$ is obtained for the roof boundary sides 4 and 8. The average $\mathrm{CI}$ (excluding the false negatives related to the roof boundary sides $11,13,15$, and 17 , for which $\mathrm{CI}=0 \%$ ) for the whole grouping of the extracted straight lines reaches $85 \%$.

Fig. 12 shows a test area with six buildings. Buildings A and D show each one the traditional hip roof, with four planar faces delimited by four roof boundary sides and five roof ridgelines. Building B shows a much more complex roof with many faces delimited by 13 roof boundary sides and 16 roof ridgelines. Note that the branch of the roof that resembles a cross-hipped roof is zoomed for facilitating the visualization of the roof details. Building $\mathrm{C}$ shows a gable roof with a small detail along one of its roof boundary sides, resulting in two planar faces delimited by 10 roof boundary sides and 1 roof ridgeline. Buildings $\mathrm{E}$ and $\mathrm{F}$ show two flat roof, delimited each one by four roof boundary sides.

Fig. 12(a) depicts the projected ALS-based roof polyhedron overlaid on the image, on which most of the roof boundary sides and ridgelines show visible registration errors. However, building B shows larger displacements along the roof boundary sides 3, 4, 6, and 20 and along the roof ridgelines 12 and 15 . Straight lines that are extracted by using the image preprocessing techniques proposed in Section II-A are overlaid on the image, as follows [see Fig. 12(b)]: Building A-13 candidate straight lines were extracted ( 1 for the roof boundary sides 1 , 2 , and 3 and for the roof ridgelines 6 and 8; and 2 for the roof boundary side 4 and for the roof ridgelines 5, 7, and 9); Building B-36 candidate straight lines were extracted ( 2 for the roof boundary sides $5,7,16$, and 17 and for the roof ridgelines 8 , $9,22,23,25$, and 26; and 1 for the remaining roof boundary sides and ridgelines); Building $\mathrm{C}-18$ candidate straight lines were extracted ( 3 for the roof boundary side 9 and for the roof ridgeline 11; 2 for roof boundary sides 4, 5, and 10; and 1 for the remaining roof boundary sides); Building D-12 candidate straight lines were extracted ( 2 for all roof boundary sides; and 1 for all roof ridgelines); Building $\mathrm{E}-8$ candidate straight lines were extracted ( 1 for the roof boundary side 3; 4 for roof boundary side 2 ; and 1 for the remaining roof boundary sides); Building $\mathrm{F}-7$ candidate straight lines were extracted ( 3 for the roof boundary side 1; 2 for the roof boundary side 2 ; and 1 for the remaining roof boundary sides). It is worth noting that a few candidates are extracted by the image processing tools proposed in Section II-A for representing the roof boundary sides and ridgelines and, in addition, in all cases, the corrected candidate 


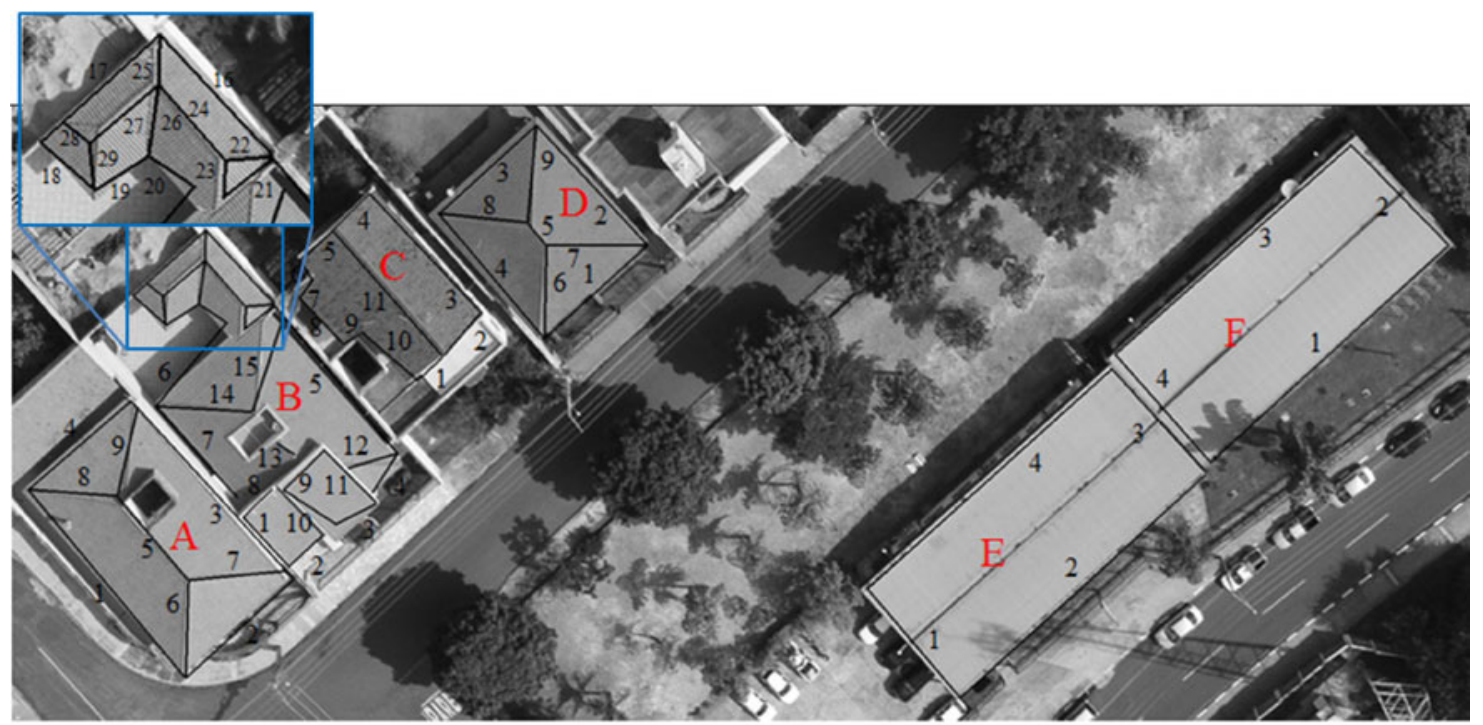

(a)

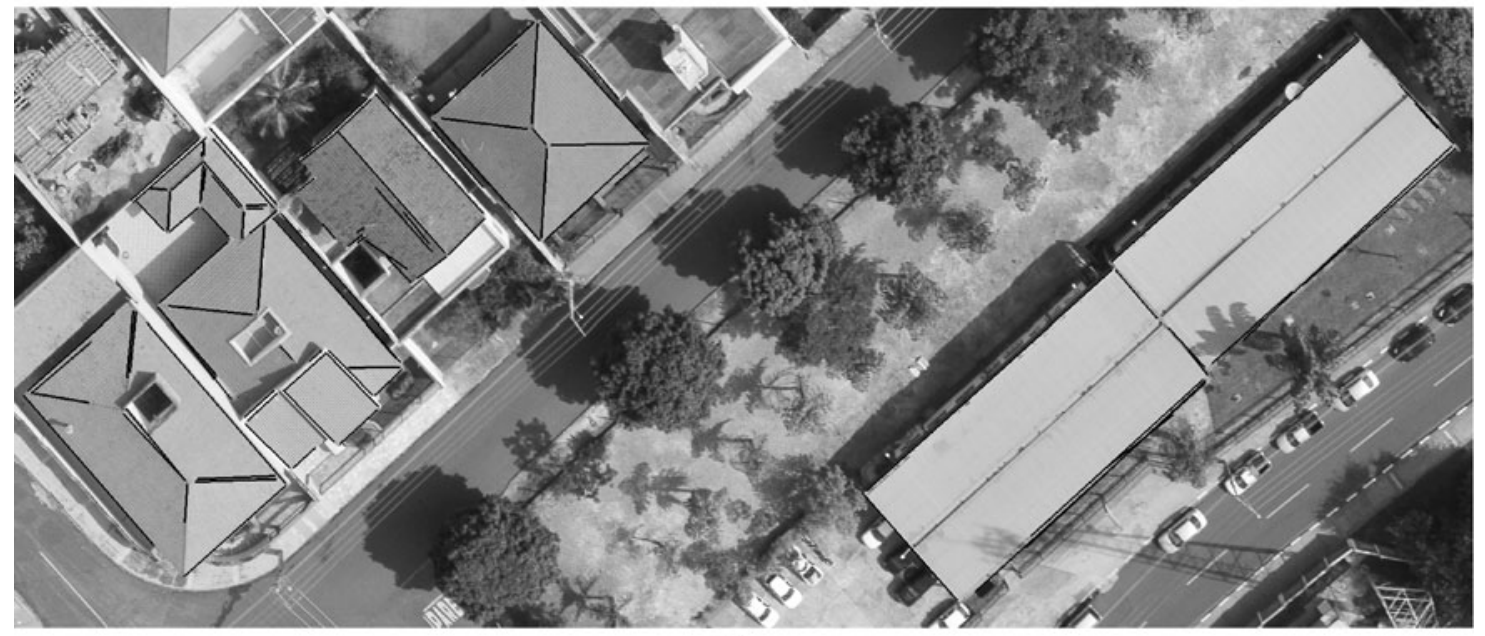

(b)

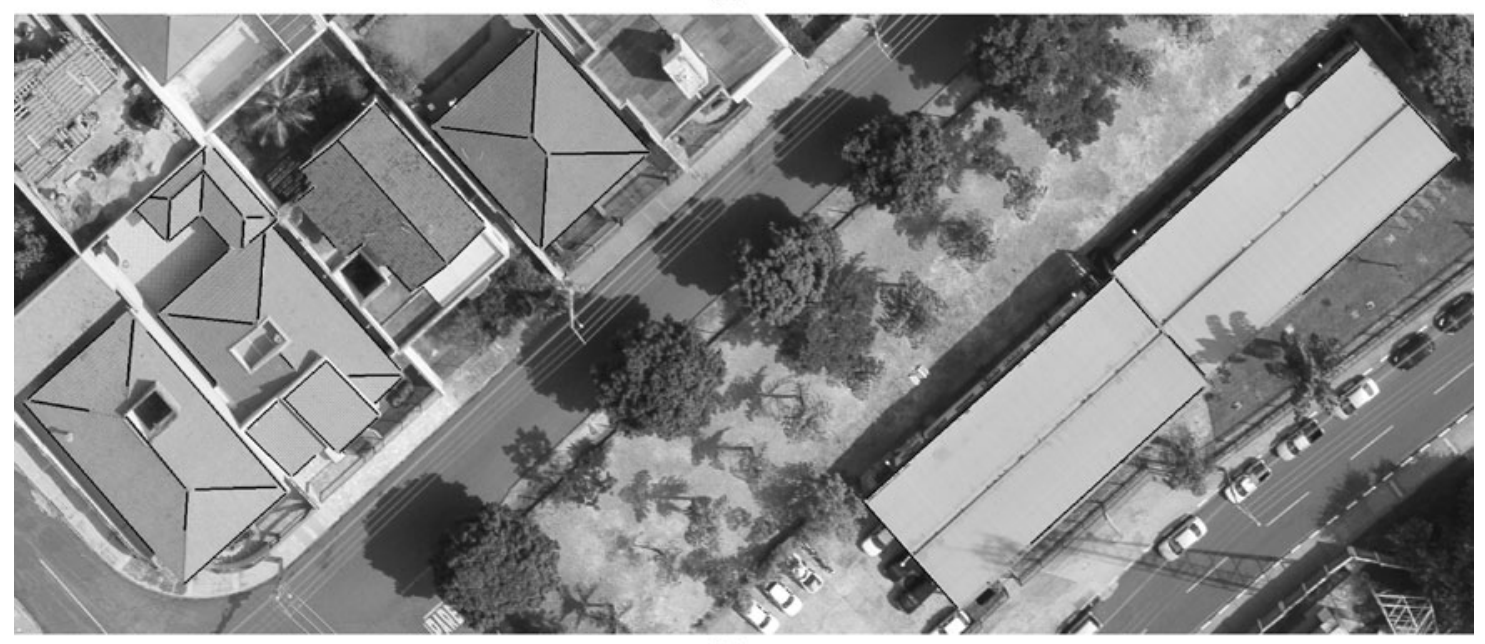

(c)

Fig. 12. Test 4. (a) Projected roofs; (b) extracted lines of the image; and (c) results of the energy function optimization. 
TABLE I

ACCURACY IMPROVEMENTS (AI)—TEST 4

\begin{tabular}{lccccc}
\hline \hline Buildings & Max. $\mathrm{AI}^{(*)}$ & $\mathrm{ESL}^{(* *)}$ & $\mathrm{Min.} \mathrm{AI}^{(*)}$ & $\mathrm{ESL}^{(* *)}$ & $\mathrm{Avg}^{(*)}$ \\
\hline $\mathrm{A}$ & 8 & 8 & 1 & 5 & 4 \\
$\mathrm{~B}$ & 11 & 3,15 & 1 & 2,18 & 5 \\
$\mathrm{C}$ & 8 & 7 & 1 & $4,8,9$ & 4 \\
$\mathrm{D}$ & 10 & 3 & 1 & 5 & 5 \\
$\mathrm{E}$ & 5 & 2 & 2 & 3 & 3 \\
$\mathrm{~F}$ & 10 & 8 & 2 & 5,7 & 5 \\
\hline \hline
\end{tabular}

${ }^{(*)}$ in pixels; ${ }^{(* *)}$ ESL: Extract straight lines

TABLE II

COMPLETION RATIO (R_LS)—TEST 4.

\begin{tabular}{lccccc}
\hline \hline Buildings & Max. CI (\%) & ESL & Min. CI (\%) & ESL & Avg CI (\%) \\
\hline A & 98 & 2 & 42 & 6 & 82 \\
B & 100 & 9 & 62 & 23 & 89 \\
C & 100 & $2,5,7$ & 23 & 6 & 68 \\
D & 98 & 7 & 86 & 5 & 93 \\
E & 100 & 1,4 & 46 & 3 & 80 \\
F & 100 & 7 & 40 & 8 & 65 \\
\hline \hline
\end{tabular}

is among the candidates for representing a roof boundary side or ridgeline. The results obtained from the application of the GA optimization algorithm are depicted in Fig. 12(c). All groupings of straight lines are corrected, i.e., no false positives and/or false negatives are verified in this experiment.

In order to quantitatively assess the results obtained in terms of accuracy improvement, the minimum (fourth column), maximum (second column), and average (sixth column) AIs for each building (first column) are computed and organized as shown in Table I. Third and fifth columns identify the extract straight lines (ESL) with maximum and minimum accuracy improvement by the number of the corresponding roof boundary sides or ridgelines. These AIs show that the lower improvements are around 1 pixel for all buildings and the higher ones are around 10 pixels, excepting for the building E. In average, the accuracy improvement is around 4 pixels.

Table II shows the minimum and maximum CIs obtained for the extracted straight lines that represent roof boundary sides and ridgelines of all buildings, besides the average CI for each building. A simple visual inspection of the Fig. 12(a) reveals that most of the straight lines are extracted with at least $90 \%$ CI and many of them reach $98 \%-100 \%$ CIs. However, few straight lines get CIs below 50\%, e.g., the straight lines 6 (buildings A and C), 3 (building E), and 8 (building F). The average CIs are above $80 \%$, excepting for buildings $\mathrm{C}$ and $\mathrm{F}$.

It is worthy commenting that the computational time is not a critical aspect of the proposed method. The basic reason is that the proposed method is individually applied to each building roof. It took longer (about $30 \mathrm{~s}$ ) for the third experiment (see Fig. 11), just because the roof has 40 roof boundary sides and ridgelines that impact the GA optimization step.

\section{Sensitivity Analysis of the Energy Function Coefficients}

The coefficients $k_{1}, k_{2}, k_{3}, k_{4}$, and $k_{5}$ provide the relative worth to the relative length, proximity, orientation, rectangularity, and corner energy terms, respectively. They should vary over $[0 ; 1]$ and their sum should be one. Based on our experience in setting these parameters for a large set of experiments, they should be set to similar values. This means that the excessive enhancement of the effect of a specific energy term should be avoided. Observing these general rules, the sensitivity of the GA optimization process in function of the changing of energy function coefficient is not critical. Moreover, these coefficients can be maintained constant in different tests, as shown in the four tests presented above. In order to illustrate the effect of the parameter changing, let us consider the building $\mathrm{C}$ of test 4 (see Fig. 12) and the following coefficient configurations: $\left(\mathrm{k}_{1}=\mathrm{k}_{2}=\mathrm{k}_{3}=\right.$ $\left.\mathrm{k}_{4}=\mathrm{k}_{5}=0.2\right) ;\left(k_{2}=0.1, k_{3}=0.3, k_{1}=k_{4}=k_{5}=0.2\right) ;$ $\left(k_{2}=0.1, k_{5}=0.3, k_{1}=k_{3}=k_{4}=0.2\right) ;\left(k_{1}=k_{2}=0.3\right.$, $k_{3}=k_{4}=0.1, k_{5}=0.2$ ). These configurations do not use any coefficient with a very different value. As expected from our experience, the results are exactly the same depicted in Fig. 12(c), which is also obtained by using the configuration $\left(k_{2}=0.1, k_{4}=0.3, k_{1}=k_{3}=k_{5}=0.2\right)$-as stated in Section III-A. Even if we enhance the relative length term with the configurations $\left(k_{1}=0.5, k_{3}=0.2, k_{2}=k_{4}=k_{5}=0.1\right.$ ) and $\left(k_{1}=0.6, k_{2}=k_{3}=k_{4}=k_{5}=0.1\right)$, the orientation term with the configurations $\left(k_{1}=0.3, k_{3}=0.4, k_{2}=k_{4}=\right.$ $\left.k_{5}=0.1\right),\left(k_{1}=0.2, k_{3}=0.5, k_{2}=k_{4}=k_{5}=0.1\right)$, and $\left(k_{3}=0.6, k_{1}=k_{2}=k_{4}=k_{5}=0.1\right)$, the rectangularity term with the configuration $\left(k_{1}=k_{5}=0.2, k_{2}=k_{3}=0.1, k_{4}=\right.$ $0.4)$, and the corner term with the configuration $\left(k_{1}=k_{4}=\right.$ $0.2 ; k_{2}=k_{3}=0.1 ; k_{5}=0.4$ ), the result remains unchanged [see Fig. 12(c)].

However, Fig. 13 shows three examples for the same building, for which wrong matches are found. The enhancement of the relative length term with a high value for $k_{1}(0.7$ or $70 \%)$ results in one wrong match (a longer straight line is select-see Figs. 12(c) and 13(a)). Fig. 13(b) shows that the enhancement of the proximity term $\left(k_{2}=0.5\right.$ or $\left.50 \%\right)$ provokes two wrong matches. As shown in Fig. 13(c), the enhancement of the orientation term with $k_{3}=0.7$ (or 70\%) results in two wrong match. Note that [see Fig. 13(c)] the longer straight line was replaced by a shorter straight line, because its orientation in relation to the neighbor projected straight line is more favorable and $k_{2}$ is set to a very low value (10\%).

\section{CONCLUSION AND FUTURE WORK}

This paper proposes a method for extracting groups of straight lines that represent roof boundaries and roof ridgelines from high-resolution aerial images using corresponding ALS-derived roof polyhedrons as initial approximations. First, a strategy for extracting straight lines around roof boundaries and ridgelines was presented, which was aimed at minimizing fragmentation and false positives. The best groups of straight lines that represent roof boundaries and ridgelines were then identified using the GA optimization method.

To demonstrate the performance of the proposed method, four experiments were presented, and their results were analyzed. The first two experiments were performed with two relatively 


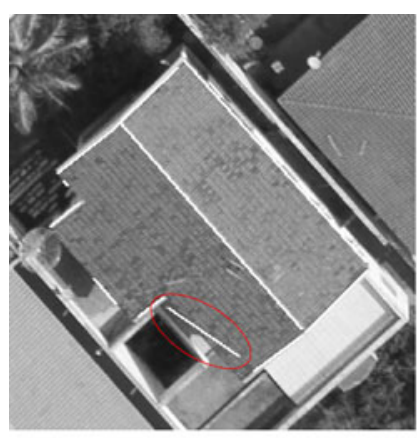

(a)

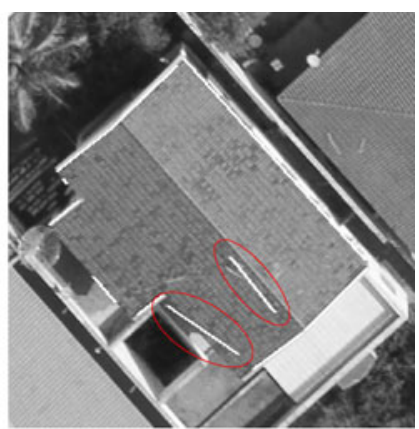

(b)

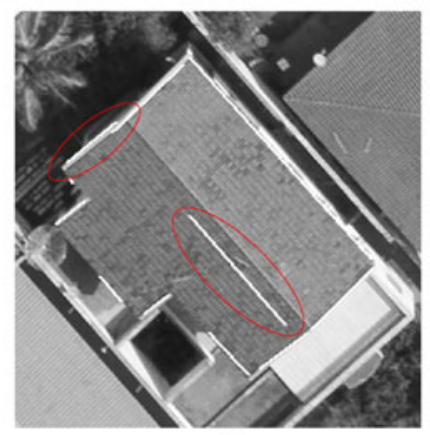

(c)

Fig. 13. Results obtained for building $\mathrm{C}$ using different coefficient configurations. (a) $k_{1}=0.7 ; k_{2}=k_{3}=k_{4}=0.1 ; k_{5}=0.0$; (b) $k_{1}=0.2 ; k_{2}=$ $0.5 ; k_{3}=k_{4}=k_{5}=0.1 ;$ e (c) $k_{3}=0.7 ; k_{1}=k_{2}=k_{4}=0.1 ; k_{5}=0.0$

simple hip roofs. The main difficulty of these examples is the relatively greater registration errors because it results in a higher chance of extracting candidates that belong to neighboring objects. Additionally, the worse the image orientation parameters, the poorer the projected ALS-based roof polyhedron, which will provide a weak reference for establishing the MRF building model. The examples showed that the proposed method can identify straight-line segments that represent roof features even when the registration error is large. All of the correspondences were correctly found for both test buildings. The third experiment was performed with a much more complex roof structure. The relatively small registration error helped to reduce the complexity of the solution space. As a result, a high success rate (36 of 40 straight lines-90\% - were correctly matched to the corresponding roof boundary sides or ridgelines) was obtained for this roof. The fourth experiment was based on a test area with six buildings of different complexities. Even though different registration errors of the projected ALS-derived roof polyhedrons are observed on the building roofs, all the correspondences are correctly found. In general, taking in consideration all experiments, no false positive or false negative is verified, excepting for experiment 3, for which four false negatives were verified. Concerning the obtained accuracies, they are widely dependent on the registration error of the corresponding projected ALSderived roof polyhedron. The obtained results showed that the accuracies of the extracted straight lines are the same or for most cases better than the corresponding projected straight lines. The obtained results also showed that a high completion ration was obtained, because the average completion ration is above $80 \%$ for most cases.
We also accomplished a sensibility analysis of the energy function coefficients. Based on our experience in setting these coefficients and on the experimental evidences presented in Section III-C, we have found that this is not a critical problem. As a general rule, it is pointed out that the coefficients should be set to similar values, i.e., it is recommended to avoid the excessive enhancement of any energy term. Moreover, they can be kept constant in all experiments.

Future work includes the use of straight lines that represent roof boundary sides, which were precisely extracted by using the proposed approach, to improve the accuracy of ALS-derived building polyhedrons. This can be accomplished by properly back-projecting the straight lines extracted by the proposed method onto the ALS-derived building polyhedrons. Another possibility for future work might be the use of the proposed method to automatically match straight lines extracted from UAV images to straight lines that define edges of high quality roof polyhedrons, and then the use of the corresponding straight lines for refining the orientation parameters of the UAV images. This might be useful whenever the exterior orientation parameters are not accurately determined during the flight mission using the integrated GPS-inertial systems.

\section{REFERENCES}

[1] S. A. N. Gilani, M. Awrangjeb, and G. Lu., "Fusion of LiDAR data and multispectral imagery for effective building detection based on graph and connected component analysis," in Proc. Int. Archives Photogramm. Remote Sens. Spatial Inf. Sci., Munich, Germany, 2015, vol. XL-3/W2, pp. 65-72.

[2] P. Fua and A. J. Hanson, "Resegmentation using generic shape: Locating general cultural objects," Pattern Recog. Lett., vol. 5, pp. 243-252, 1987.

[3] D. S. Müller and W. Zaum, "Robust building detection in aerial images," in Proc. Int. Archives Photogramm. Remote Sens. Spatial Inf. Sci., Vienna, Austria, 2005, vol. 36-3/W24, pp. 143-148.

[4] H. G. Akçay and S. Aksoy, "Automatic detection of geospatial objects using multiple hierarchical segmentations," IEEE Trans. Geosci. Remote Sens., vol. 46, no. 7, pp. 2097-2111, Jul. 2008.

[5] G. Ferraioli, "Multichannel InSAR building edge detection," IEEE Trans. Geosci. Remote Sens., vol. 48, no. 3, pp. 1224-1231, Mar. 2010.

[6] B. Sirmaçek and C. A. Ünsalan, "Probabilistic framework to detect buildings in aerial and satellite images," IEEE Trans. Geosci. Remote Sens., vol. 49, no. 1, pp. 211-221, Jan. 2011.

[7] T. V. Yamazaki and F. M. Matsuoka, "Multi-scale solution for building extraction from LiDAR and image data," Int. J. Appl. Earth Observ. Geoinf., vol. 11, no. 4, pp. 281-289, 2009.

[8] Y. Li and H. Wu, "An improved building boundary extraction algorithm based on fusion of optical imagery and LiDAR data," Optik, vol. 124, no. 22, pp. 5357-5362, 2013.

[9] L. Matikainen, J. Hyyppä, and H. Hyyppä, "Automatic detection of buildings from laser scanner data for map updating," in Proc. Int. Archives Photogramm. Remote Sens. Spatial Inf. Sci., Dresden, Germany, 2003, vol. 34 .

[10] D. Tóvari and T. Vögtle, "Object classification in laserscanning data," in Proc. Int. Archives Photogramm. Remote Sens. Spatial Inf. Sci., Istanbul, Turkey, 2004, vol. 36-8/W2, pp. 45-49.

[11] F. Tarsha-Kurdi, T. Landes, P. Grussenmeyer, and E. Smigiel, "New approach for automatic detection of buildings in airborne laser scanner data using first echo only," in Proc. Int. Archives Photogramm. Remote Sens. Spatial Inf. Sci., Bonn, Germany, 2006, vol. 36, pp. 25-30.

[12] A. Sampath and J. Shan, "Building boundary tracing and regularization from airborne LiDAR point clouds," Photogrammetric Eng. Remote Sens., vol. 73, no. 7, pp. 805-812, 2007.

[13] S. Wei, "Building boundary extraction based on lidar point clouds data," in Proc. Int. Archives Photogramm. Remote Sens. Spatial Inf. Sci., Beijing, China, 2008, vol. 37, Part B3b, pp. 157-162. 
[14] S. N. Perera, H. A. Nalani, and H. Maas, "An automated method for 3D roof outline generation and regularization in airbone laser scanner data," in Proc. Int. Archives Photogramm. Remote Sens. Spatial Inform. Sci., Melbourne, Australia, 2012, vol. I-3, pp. 281-286.

[15] F. Rottensteiner, J. Trinder, S. Clode, and K. Kubik, "Automated delineation of roof planes from LiDAR Data," in Proc. Int. Archives Photogramm. Remote Sens. Spatial Inf. Sci., Vienna, Austria, 2005, vol. 363/W19, pp. 221-226.

[16] A. Sampath and J. Shan, "Segmentation and reconstruction of polyhedral building roofs from aerial LiDAR point clouds," IEEE Trans. Geosci. Remote Sens., vol. 48, no. 3, pp. 1554-1621, Mar. 2010.

[17] A. Henn, G. Groger, V. Stroh, and L. Plumer, "Model driven reconstruction of roofs from sparse LIDAR point clouds," ISPRS J. Photogramm. Remote Sens., vol. 76, pp. 17-29, 2013.

[18] Y. Chen, L. Cheng, M. Li, J. Wang, L. Tong, and K. Yang, "Multiscale grid method for detection and reconstruction of building roofs from airborne LiDAR data," IEEE J. Select. Topics Appl. Earth Observ. Remote Sens., vol. 7, no. 10, pp. 4081-4094, Oct. 2014

[19] N. Haala and C. Brenner, "Extraction of buildings and trees in urban environments," ISPRS J. Photogramm. Remote Sens., vol. 54, pp. 130137, 1999.

[20] G. Sohn and I. J. Dowman, "Building extraction using lidar DEMs and ikonos images," in Proc. Int. Archives Photogramm. Remote Sens. Spatial Inf. Sci., Dresden, Germany, 2003, vol. 34.

[21] G. Vosselman, "Fusion of laser scanning data, maps, and aerial photographs for building reconstruction," in Proc. IEEE Int. Symp. Geosci. Remote Sens., Toronto, Canada, 2002, vol. 1, pp. 85-88.

[22] J. J. Jaw and C. C. Cheng, "Building roof reconstruction by fusing laser range data and aerial images," in Proc. Int. Archives Photogramm. Remote Sens. Spatial Inf. Sci., Beijing, China, 2008, vol. XXXVII-B3b, pp. 707-712.

[23] A. P. Dal Poz, "Geometric refinement of LiDAR roof contours using photogrammetric data and Markov random field model," in Proc. Int. Archives Photogramm. Remote Sens. Spatial Inf. Sci., Beijing, China, 2008, vol. XXXVII-B4, pp. 427-432.

[24] C. Kim and A. Habib, "Object-based integration of photogrammetric and lidar data for automated generation of complex polyhedral building models," Sensor, vol. 9, pp. 5679-5701, 2009.

[25] L. Chen and S. Zhao, "Building detection in an urban area using LiDAR data and quickbird imagery," Int. J. Remote Sens., vol. 33, no. 15, pp. 51355148,2012

[26] L. Cheng et al., "Integration of LiDAR data and optical multi-view images for 3D reconstruction of building roofs," Opt. Lasers Eng., vol. 51, pp. 493-502, 2013.

[27] G. Zhou and X. Zhou, "Seamless fusion of LiDAR and aerial imagery for building extraction," IEEE Trans. Geosci. Remote Sens., vol. 52, no. 11, pp. 7393-7407, Nov. 2014.

[28] A. Zarea and A. Mohammadzadeh, "A novel building and tree detection method from LiDAR data and aerial images," IEEE J. Sel. Topics Appl. Earth Observ. Remote Sens., vol. 9, no. 5, pp. 1864-1875, May 2016.
[29] M. Awrangjeb, C. Zhang, and C. S. Fraser, "Automatic extraction of building roofs using LIDAR data and multispectral imagery," ISPRS J. Photogramm. Remote Sens., vol. 83, pp. 1-18, 2013.

[30] P. R. Wolf and C. D. Ghilani. Adjustment Computations: Statistics and Least Squares in Surveying and GIS, 3rd ed. New York, NY, USA: Wiley, 1997.

[31] J. Canny, "A computational approach to edge detection," IEEE Trans Pattern Anal. Mach. Intell., vol. PAMI-8, no. 6, pp. 679-698, 1986.

[32] C. Steger, "Extracting lines using differential geometry and gaussian smoothing," in Proc. Int. Archives Photogramm. Remote Sens., Vienna, Austria, 1996, vol. 31-B3, pp. 821-826.

[33] R. Jain, R. Kasturi, and B. G. Schunck, Machine Vision (Computer Science Series). New York, NY, USA: MIT Press and McGraw-Hill, 1995.

[34] D. H. Douglas and T. K. Peucker, "Algorithms for the reduction of the number of points required to represent a digitized line or its caricature," Can. Cartographer, vol. 10, no. 2, pp. 112-122, 1973.

[35] S. K. Kopparapu and U. B. Desai, Bayesian Approach to Image Interpretation. Boston, MA, USA: Kluwer, 2001.

[36] D. E. Goldberg, Genetic Algorithms in Search, Optimization, and Machine Learning. Reading, MA, USA: Addison-Wesley, 1989.

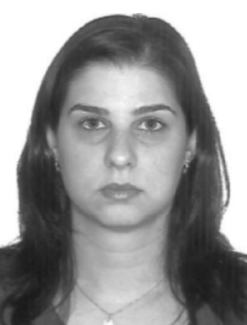

Vanessa Jordão Marcato Fernandes received the B.Sc. degree in cartographic engineering and the M.Sc. degree in cartographic sciences, in 2011 and 2013, respectively, from São Paulo State University, Presidente Prudente, Brazil, where she is currently working toward the Ph.D. degree in the graduate program in cartographic sciences.

Her research interests include remote sensing and image analysis.

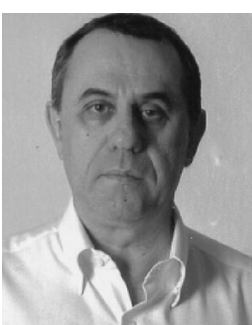

Aluir Porfírio Dal Poz received the B.Sc. degree in cartographic engineering from São Paulo State University, Presidente Prudente, Brazil, in 1987, the M.Sc. degree in geodetic science from Paraná Federal University, Curitiba, Brazil, in 1991, and the Ph.D degree in transportation engineering from São Paulo University in 1996.

He is currently a Full Professor in the Department of Cartography, São Paulo State University. His expertise and current research interests include the areas of image analysis and digital photogrammetry. 